\title{
Unveiling True Three-dimensional Microstructural Evolution in Novel Chalcogenide Nanocomposites as a Route to Infrared Gradient Refractive Index Functionality
}

Myungkoo Kang ${ }^{1}$, Hugues Francois-Saint-Cyr ${ }^{2}$, Isabelle Martin ${ }^{2}$, Rashi Sharma ${ }^{1}$, Cesar Blanco ${ }^{1}$, Stoichko Antonov ${ }^{3}$, Ty Prosa ${ }^{2}$ and Kathleen Richardson ${ }^{1}$

${ }^{1}$ University of Central Florida, Orlando, Florida, United States, ${ }^{2}$ CAMECA Instruments Inc., Madison, Wisconsin, United States, ${ }^{3}$ Max-Planck-Intitut fur Eisenforschung GmbH, Dusseldorf, NordrheinWestfalen, Germany

Infrared (IR) components based on spherical or aspherical lenses have been widely used in imaging systems. However, legacy IR systems are large and heavy due to multiple crystalline optical components required for compensation of chromatic aberration. Gradient refractive index (GRIN) materials have been explored as a potential solution to this challenge [1]. Among candidate materials for GRIN, Ge-As-Pb-Se (GAP-Se)-based chalcogenide glasses have received interest due to the ability to modulate their refractive index $(n)$ through the formation of high- $n$ nanocrystals within the glassy matrix upon heat treatment, while maintaining their broadband infrared transparency. The effective $n$ of the resulting glass-ceramic nanocomposites increases with the volume fraction $(V)$ of the high- $n$ nanocrystals. The glass-ceramic nanocomposite's effective $n$ can be approximated by the summation of the $n \times V$ product for induced crystal phases and a residual amorphous matrix. The formulation indicates that spatial variation in the volume fraction of high- $n$ crystals leads to an effective medium with a gradient in both $n$ and spectral dispersion [1,2].

Transmission electron microscopy (TEM) data previously collected from $\mathrm{GeSe}_{2}-\mathrm{As}_{2} \mathrm{Se}_{3}-\mathrm{PbSe}$ base glasses indicate that the glasses made upon a melt-quench process exhibit composition-dependent morphological variations [3]. Specifically, an immiscibility zone has been reported to exist for compositions with $\sim 10$ to $\sim 50 \mathrm{~mol} \%$ PbSe where an increase in PbSe content involves morphological transitions from $\mathrm{Pb}$-rich particles in a $\mathrm{Pb}$-deficient matrix $(\sim 10-\sim 25 \mathrm{~mol} \%)$ to a spinodal decompositionlike structure $(\sim 25-\sim 35 \mathrm{~mol} \%)$ to $\mathrm{Pb}$-deficient particles in a Pb-rich matrix $(\sim 35 \sim \sim 50 \mathrm{~mol} \%)$. Interestingly, heat treatment makes the energetically unstable Pb-rich phases exclusively crystallized while keeping the $\mathrm{Pb}$-deficient phases amorphous. This phenomenon is of particular importance since the $\mathrm{Pb}$-rich crystallites formed upon heat treatment have a value of $n$ far greater than that of the $\mathrm{Pb}$-deficient phase, thereby leading to an increase in effective $n$ of a resulting glass-ceramic nanocomposite. However, the quantitative information such as their size, shape, connectivity, and composition of the co-existing phases has remained unclear, due to limitations in a two-dimensional (2-D) perspective of TEM. Meanwhile, atom probe tomography (APT) is a promising complementary technique, providing a 3-D reconstruction of constituent atoms' spatial distribution from a specimen of interest. Here, we show an ongoing progress towards the accurate quantification of such information in a 3-D space for three compositions with 20,30 , and $40 \mathrm{~mol} \% \mathrm{PbSe}$ each of which represents the three morphological domains in the immiscibility zone.

Two identical sets of $20 \mathrm{GeSe}_{2}-60 \mathrm{As}_{2} \mathrm{Se}_{3}-20 \mathrm{PbSe}, 17.5 \mathrm{GeSe}_{2}-52.5 \mathrm{As}_{2} \mathrm{Se}_{3}-30 \mathrm{PbSe}$, and 15GeSe2$45 \mathrm{As}_{2} \mathrm{Se}_{3}-40 \mathrm{PbSe}$ bulk glasses were prepared using a standard melt-quench technique [3]. Subsequently, one of the sets underwent heat treatment to convert those into glass-ceramics [3]. Focused ion beam (FIB)assisted milling was then performed on the bulk glasses to prepare APT specimens of a needle shape with 
100nm tip radius using FEI Helios Nanolab 660 FIB-SEM. Finally, the microstructure of the specimens was three-dimensionally reconstructed from the APT analysis using a CAMECA LEAP 5000XR. It is important to note that these particular chalcogenide glasses and glass-ceramics are substantially sensitive to irradiation, leading to excessively fast milling. Therefore, it took time to develop a procedure to prepare specimens with the FIB and optimize a set of analytical parameters to acquire good results with the atom probe. Specifically, we have empirically identified a set of optimal process conditions, including the deposition of a sacrificial Ni layer prior to the FIB-assisted milling as well as low base temperature of $30 \mathrm{~K}$, high detection rate of $2 \%$, low pulse frequency of $65 \mathrm{kHz}$, and extremely low laser pulse energy of 10 - 200fJ for the APT data collection. These conditions led to excellent mass resolving power of mass spectra in the order of 1000 which enabled the accurate identification of constituent elements and their compounds [4].

Figure 1 shows scanning electron microscopy (SEM) images of APT tips, 3-D ion maps of the tips, and proximity histograms of constituent elements across multiple particle-matrix interfaces for both base glasses and glass-ceramics with 20,30 , and $40 \mathrm{~mol} \% \mathrm{PbSe}$. There are four key findings observed from the data. First, the SEM images of APT tips and their corresponding 3-D ion maps where the particle-matrix boundaries are determined based on isoconcentration surfaces of $5 \mathrm{at} \% \mathrm{~Pb}$ show that there are $\mathrm{Pb}$-rich particles within a $\mathrm{Pb}$-deficient matrix for the composition with $20 \mathrm{~mol} \% \mathrm{PbSe}$. On the other hand, the glass with $40 \mathrm{~mol} \% \mathrm{PbSe}$ shows an inverse configuration where $\mathrm{Pb}$-deficient particles are distributed within a $\mathrm{Pb}$-rich matrix. The intermediate composition with $30 \mathrm{~mol} \% \mathrm{PbSe}$ shows an interwoven structure of $\mathrm{Pb}$ rich and $\mathrm{Pb}$-deficient phases typical of spinodal decomposition. Second, in all cases, the proximity histograms show that $\mathrm{As}$ and $\mathrm{Se}$ are deficient within locations where $\mathrm{Pb}$ is rich, while the spatial distribution of $\mathrm{Ge}$ is relatively constant. Third, these histograms also show that the atomic percentages of constituent elements remain very similar upon heat treatment, indicating no long-range atomic diffusion during the crystallization process. Fourth, the development of facets at the particle-matrix interfaces in glass-ceramics indicates the presence of crystallites which emerge as a consequence of heat treatment. To statistically quantify the size, shape, and volume fraction of the particles, endeavors to collect data from multiple regions of interest are currently in progress where further insight into the role of the 3-D microstructures in a process-structure-property relationship for this material system would be revealed $[5]$.
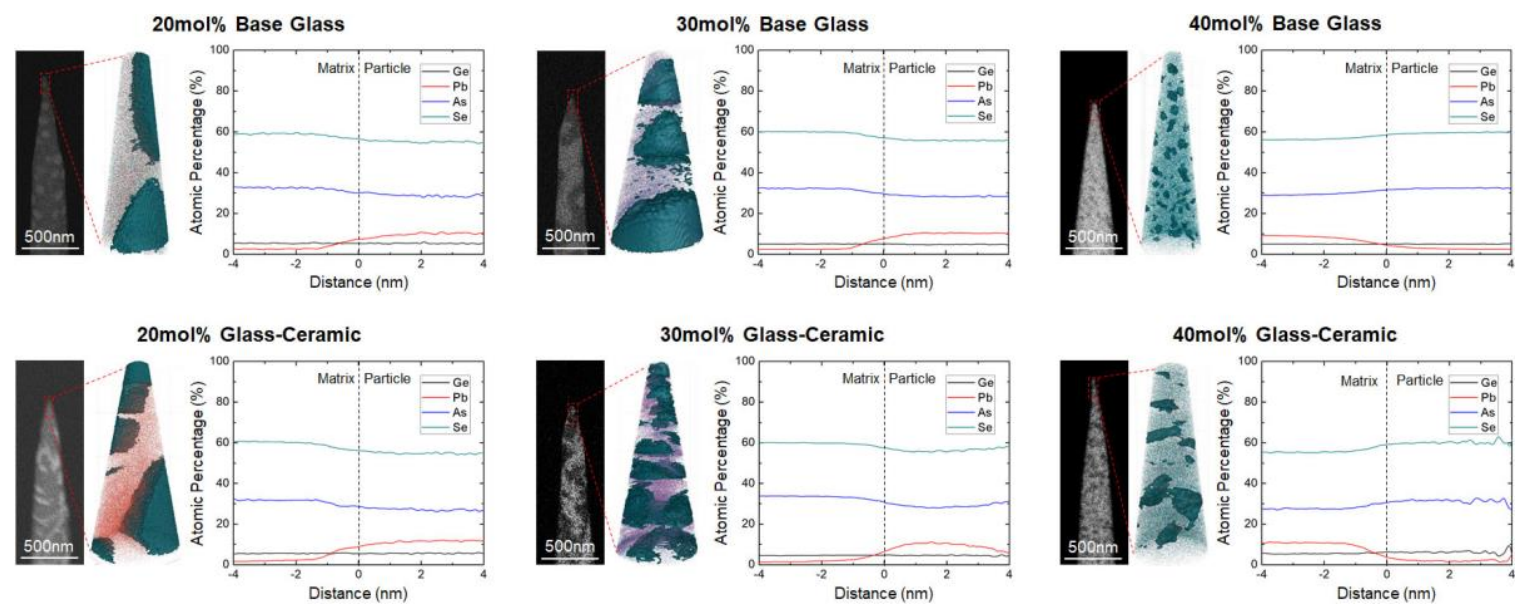

Figure 1. SEM images of APT tips, 3-D ion maps, and proximity histograms of constituent elements for base glasses and glass-ceramics with 20, 30, and 40mol\% $\mathrm{PbSe}$. 


\section{References}

[1] J. Teichman, J. Holzer, B. Balko, B. Fisher, L. Buckley, Gradient Index Optics at DARPA, The Institute for Defense Analyses, Alexandria, VA, USA 2014.

[2] M. Kang, A. M. Swisher, A. V. Pogrebnyanov, L. Liu, A. Kirk, S. Aiken, L. Sisken, C. Lonergan, J. Cook, T. Malendevych, F. Kompan, I. Divliansky, L. B. Glebov, M. C. Richardson, C. Rivero-Baleine, C. G. Pantano, T. S. Mayer*, and K. Richardson, "Ultra-low Dispersion Multicomponent Thin Film Chalcogenide Glass for Broadband Gradient Index Optics," Adv. Mater. 30, 1803628 (2018).

[3] L. Sisken, M. Kang, J. M. Veras, C. Smith, A. Buff, A. Yadav, D. McClane, C. Blanco, C. RiveroBaleine, T. S. Mayer, and K. Richardson, "Infrared Glass Ceramics with Multi-Dispersion and Gradient Refractive Index Attributes,” Adv. Funct. Mater. 29, 1902217 (2019).

[4] H. Francois-Saint-Cyr, M. Kang, I. Martin, S. Antonov, T. J. Prosa, and K. Richardson, "ThreeDimensional Microstructural Characterization of Novel Chalcogenide Nanocomposites for Gradient Refractive Index Applications," Microsc. Microanal. 25, 2500 (2019).

[5] The authors acknowledge the partial support of UCF's Pre-eminent Post-doctoral Scholar Program. 